\title{
Análisis de las tomas de decisión en la fase de ataque de las jugadoras aleros de baloncesto. \\ Analysis of decision-making when female basketball forguard players are in offense.
}

\author{
Ana Concepción Jiménez Sánchez \\ Facultad de Ciencias de la Actividad Física y el Deporte-INEF \\ Universidad Politécnica de Madrid \\ Luis Miguel Ruiz Pérez \\ Facultad de Ciencias del Deporte \\ Universidad de Castilla-La Mancha. Toledo
}

\section{R e s u m e n}

En este texto se describe la investigación realizada por los autores, ante la inquietud por conocer qué tipo de tomas de decisión tienen las jugadoras de alto nivel en baloncesto cuando se encuentran en la fase de ataque y, más concretamente, cuando se encuentran en posesión del balón. Siendo conscientes de que las situaciones de partido son aquellas en que las decisiones que toman las jugadoras pueden tener una trascendencia directa sobre el resultado final, la investigación se ha centrado en conocer a determinadas jugadoras aleros de la máxima categoría, Liga Femenina, durante la competición a través de una temporada.

Para conseguir los objetivos previstos se procedió a la realización de distintos estudios con la intención de incrementar y relacionar la información obtenida a través de éstos. De este modo tuvimos un amplio conocimiento sobre las jugadoras aleros en cuanto a sus características, funciones, antecedentes en el baloncesto, nivel de experiencia, preferencias, percepción sobre sí mismas, tipos y estilos de decisión, elementos de referencia que utilizan al decidir en el juego, acciones frecuentes durante el juego, y cuál es su pensamiento sobre las decisiones tomadas en dicha competición.
In this text the investigation carried out by the authors is described, in view of the interest in knowing which kind of decision-making have players with a basketball high level when they are attacking and, more specifically, when they are in possession of the ball.

Bearing in mind that the situations of match are those in which decision-making made by players can have a direct significance on the final score, the investigation has focused on knowing certain forward players of the highest category, Female League, in the competition during one season.

In order to achieve expected aims, the carrying out of different studies with the intention of increasing and relationating the information, got through these ones began. In this way, we had a wide knowledge about guard players characteristics, roles, records in basketball, level of experience, preferences, perception inside themselves, kinds of decision, references used when deciding during the game and frequent actions and which their knowledge about decision made in this competition is.

Palabras clave: Baloncesto, tomas de decisión, ataque, alero.

Key Words: Basketball, decision-making, offense, forward.

Correspondencia/correspondence: Facultad de Ciencias de la Actividad Física y el Deporte-INEF. Universidad Politécnica de Madrid. Calle Martín Fierro s/n 28040 Madrid (Spain)

Teléfono: 34913364109 - E-mail: anaconcepcion.jimenez@upm.es 


\section{Introducción}

En baloncesto, en el transcurso de un partido, donde el ritmo de las acciones es rápido y cambiante, es preciso percibir las situaciones que se presentan en el juego, con gran claridad. Es muy importante que las decisiones sean correctas, a expensas del breve tiempo del que se dispone, del mismo modo que las acciones tienen que ser precisas.

Las investigaciones en el deporte, relacionadas con los procesos cognitivos, han ido aportando luz y enriqueciendo la labor cualitativa de los profesionales en el área de la enseñanza y el entrenamiento del deporte, siendo los deportistas los receptores directos del conocimiento adquirido.

Los estudios de baloncesto en los últimos años han indagado sobre múltiples elementos relacionados con los procesos cognitivos, con una relación más o menos directa en las tomas de decisión. Un ejemplo de ello lo muestran Ruiz y Arruza (2005), realizando un análisis pormenorizado sobre las tomas de decisión en los deportistas y cómo optimizar este proceso en el desarrollo de los entrenamientos.

Las inquietudes por crear, conocer y aplicar modelos de enseñanza del baloncesto y técnicas que impliquen la adquisición y la mejora de las tomas de decisión, son muy relevantes, ofreciendo claridad a los docentes sobre cómo obtener mejoras durante el juego (Cárdenas y Pintor, 2001; Castejón y López, 2000; Dupré, 2001; Giménez y Sáenz-López, 2000, 2002; Hernández, 2001; Ibáñez, 2000; Olivera, 2001; Sáenz-López, 2000; Tavares, 2002; Temprado, 1989, 1992; Temprado y Alain, 1999; Temprado y Famose, 1999; Ticó, 2002).

Los trabajos relacionados con el conocimiento en baloncesto, han intentado comprobar cuál es el grado de relación entre los conocimientos declarativos y procedimentales, y cuáles son las diferencias entre chicos principiantes y aquellos más expertos (French, Nevett, Spurgeon, Graham, Rink y McPherson, 1996; French y Thomas, 1987; Temprado, 1989, 1992; Temprado y Alain, 1999; Temprado y Famose, 1999; Tenenbaum, Yuval, Elbaz, Bar-Eli y Weinberg, 1993; Thomas, French y Humphries, 1986).

En relación con los expertos, sus características; cómo toman decisiones; cómo optimizar su rendimiento y, en muchos casos, cuáles son las diferencias y similitudes con los deportistas novatos, han sido y son fuentes de investigación en baloncesto. (Abernethy, 1987, 1989; Buscá, Pont, Artero y Riera, 1996; Castejón, 2002b; French, Spurgeon y Nevett, 1995; Lorenzo, 2003; Ribas y Fraile, 2003; Ripoll, 1988; SáenzLópez, Giménez, Sierra, Ibáñez y Sánchez, 2003; Sánchez Sánchez, 2002; Tavares, 2002).

Los trabajos sobre los estilos al decidir en baloncesto, han ido orientados hacia los entrenadores, logrando determinar diferentes estilos y comprobar cuáles son más favorables para conseguir los objetivos previstos (Chelladurai, 1991; Chelladurai y Quek, 1995; Lane y Damiano, 1994). Sobre las decisiones estratégicas que toman los 
entrenadores de baloncesto, también han realizado algunos estudios interesantes (Castejón, Aguado, Cabrera, García, Gismero, Rodríguez y Romero, 2004). Sin embargo estudios actuales se centran también en los jugadores indagando sobre los diversos estilos al decidir de los propios deportistas. (Fernández, Sánchez y FernándezQuevedo, 2002; Ruiz, Graupera y Navarro, 2000).

La línea de investigación desarrollada por Refoyo (2001), valorando en qué medida ciertas decisiones tácticas de los jugadores de baloncesto, en determinadas acciones del juego, se encuentran condicionadas por el estado fisiológico de estos jugadores, aporta nuevos datos a la comprensión de estos procesos cognitivos.

Consideramos interesantes los trabajos de investigación realizados por Iglesias, Moreno, Ramos, Fuentes, Julián y Del Villar (2002), y los elaborados por Moreno, Fuentes, Del Villar, Iglesias y Julián (2003), orientados específicamente hacia el estudio y análisis de los procesos cognitivos desarrollados por los jugadores de baloncesto durante las tomas de decisión. El objetivo, es intentar determinar el pensamiento de los jugadores mientras toman las decisiones y comprobar diferencias en cuanto a las acciones ejecutadas, así como establecer comparaciones entre los dos grupos de jugadores pertenecientes a dicha investigación.

\section{Análisis de las tomas de decisión de las jugadoras aleros en posesión del balón.}

La investigación se realizó con las jugadoras aleros de la Comunidad de Madrid, pertenecientes a la Liga Femenina, cuando tenían posesión del balón en la fase de ataque, y ante una defensa individual. Fueron un total de nueve jugadoras analizadas.

Como hemos indicado, esta investigación se trató con diferentes estudios que valoraron distintas perspectivas, a través de las cuales abordamos el conocimiento de las tomas de decisión de las jugadoras. Concretamente fueron seis los estudios realizados.

\section{Objetivos de la investigación}

Los objetivos generales de la investigación fueron los siguientes:

1. Conocer a las jugadoras aleros de la Liga Femenina en el ámbito del entrenamiento y la competición de baloncesto, desde la percepción que tienen sobre sí mismas en cuanto a sus características, decisiones y acciones durante los partidos.

2. Analizar la percepción que los entrenadores tienen del puesto específico del alero y sobre las responsabilidades que éstas tienen al tomar decisiones durante los partidos.

3. Valorar la percepción del éxito que tienen las jugadoras aleros de baloncesto, como elemento que puede influir en sus tomas de decisión.

4. Detectar las preferencias de las jugadoras por un tipo de estilo al decidir en baloncesto. 
Jiménez, A.C., Ruiz, L.M. (2006). Análisis de las tomas de decisión en la fase de ataque de las jugadoras aleros de baloncesto. Revista Internacional de Ciencias del Deporte. 4(2), 26-46.

5. Analizar las diferentes acciones técnicas que realizan las jugadoras aleros de baloncesto durante los partidos, cuando reciben el balón en el campo de ataque.

6. Analizar la percepción y comprensión que las jugadoras tienen de sus propias decisiones en situaciones reales.

7. Elaborar un perfil decisional de la jugadora alero de baloncesto.

\section{Metodología}

Se optó por una metodología cualitativa y cuantitativa descriptiva, en la que a través de la aplicación de cuestionarios y entrevistas, analizamos las respuestas de las jugadoras y los entrenadores de baloncesto de alta competición femenina. Las respuestas obtenidas, se analizaron extrayendo los aspectos coincidentes más relevantes y aquellos que diferían para, de este modo, poder obtener unos resultados y elaborar unas conclusiones finales.

\section{Procedimientos}

Podríamos resumir los procedimientos enmarcados en cinco etapas:

\section{$1^{a}$ etapa. Antes de iniciar la temporada}

o Contactos y solicitud de permisos a presidentes de los clubes, entrenadores y jugadoras para realizar los estudios en cuestión.

\section{$2^{a}$ etapa. Al inicio de la competición}

o Elección y elaboración de los instrumentos adecuados.

o Realización de estudio piloto.

o Diseño sobre las pautas de la filmación de los partidos.

\section{$3^{a}$ etapa. Iniciada la competición}

o Realización de los cuestionarios de los diferentes estudios.

\section{$4^{a}$ etapa. Durante la competición}

o Filmación y análisis de los partidos.

$5^{a}$ etapa. Finalizando la competición

o Realización de las entrevistas a las jugadoras.

\section{Instrumentos}

Los instrumentos utilizados en cada uno de los estudios fueron los siguientes: 
Jiménez, A.C., Ruiz, L.M. (2006). Análisis de las tomas de decisión en la fase de ataque de las jugadoras aleros de baloncesto. Revista Internacional de Ciencias del Deporte. 4(2), 26-46.

\section{1.- Cuestionario a los entrenadores de las jugadoras aleros de baloncesto (Q.EJAB).}

Reclama las opiniones de los entrenadores sobre las cualidades de las jugadoras aleros de Baloncesto femenino de alta competición. Consta de 26 preguntas para obtener información sobre las características de las jugadoras aleros, así como sobre el papel que desempeñan en el equipo y durante los partidos.

\section{2.- Cuestionario sobre las jugadoras aleros de baloncesto (Q.JAB)}

Está compuesto por 58 preguntas que demandan aspectos relacionados con la trayectoria deportiva, características personales, conocimiento y preferencias del Baloncesto de las jugadoras aleros de los equipos de alta competición de la Comunidad de Madrid, participantes en este estudio.

\section{3.- Hoja de Análisis de Tomas de decisión en baloncesto (HATD)}

Este instrumento tiene el propósito de permitir recoger los datos observados durante los partidos. En esta hoja se recogen todas las posibles acciones de las jugadoras, así como las circunstancias que rodean a cada una de las acciones (por ejemplo, parámetros de tiempo y resultado; distancias del aro y la defensora).

4.- Cuestionario sobre percepción del éxito (Roberts y Balagué, 1991). Adaptación de Escartí y Cervelló.

Este cuestionario consta de 12 ítems que evalúan la orientación al ego o tarea de las jugadoras aleros.

5.- Cuestionario de estilo al decidir en el deporte (CED) (Ruiz, Sánchez y Graupera 1997).

Consta de 30 ítems referidos a tres dimensiones de la toma de decisión: Compromiso con el aprendizaje táctico, competencia decisional percibida y ansiedad y agobio al decidir.

6.- Entrevista evocadora sobre la comprensión del proceso decisional de las jugadoras después de los partidos

Consta de una serie de cuestiones que permiten incitar a las jugadoras a explicar sus decisiones ante el visionado de su propia actuación, dentro de un enfoque de recuerdo estimulado. 


\section{Estudios realizados en la investigación}

\section{Estudio 1}

\section{Análisis de las tomas de decisión y acción desde la perspectiva de los entrenadores}

Consideramos que era importante que todos entrenadores de los equipos de la Liga Femenina, nos ofrecieran su perspectiva de las jugadoras aleros, y que sus respuestas pudieran aportar datos relevantes para tener un mayor conocimiento de dichas jugadoras. De los doce entrenadores, contamos con la colaboración de ocho, de los cuales, tres de ellos eran entrenadores de los equipos a los que pertenecían las jugadoras investigadas.

Los objetivos de esta investigación fueron los siguientes:

1. Conocer cuál es la consideración que los entrenadores tienen del puesto de alero, y analizar el papel que éstos asignan a las jugadoras aleros en la fase de ataque.

2. Describir la concepción que tienen los entrenadores del juego de ataque, con relación a las funciones de las jugadoras aleros, así como cuáles son los niveles de responsabilidad que les asignan.

3. Conocer las características socioprofesionales de los entrenadores de la Liga Femenina de Baloncesto.

\section{Resultados}

Se aplicó el Cuestionario a los entrenadores de las jugadoras aleros de Baloncesto (Q.EJAB), de cuyo análisis de las respuestas obtuvimos unos resultados que los siguientes resultados:

\begin{tabular}{|c|c|}
\hline \multicolumn{2}{|c|}{$\begin{array}{c}\text { PERFIL DE LAS JUGADORAS ALEROS DE BALONCESTO } \\
\text { (Según los entrenadores de la Liga Femenina) }\end{array}$} \\
\hline Características de la jugadora alero & 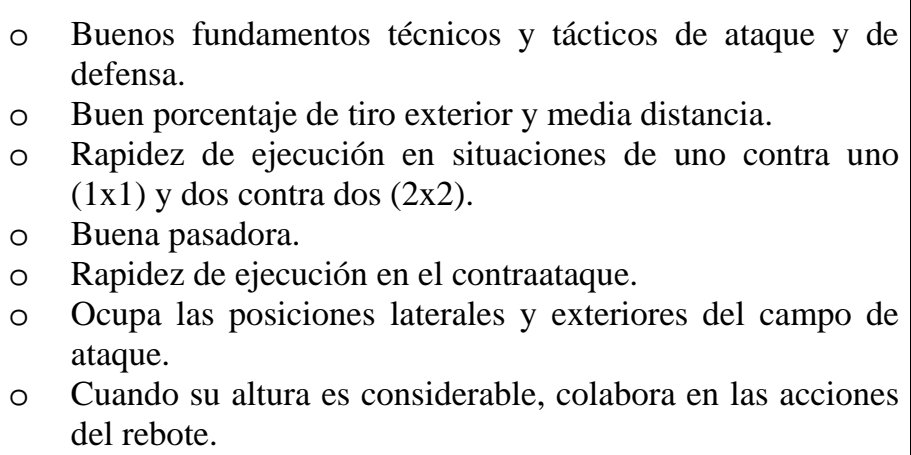 \\
\hline
\end{tabular}


Jiménez, A.C., Ruiz, L.M. (2006). Análisis de las tomas de decisión en la fase de ataque de las jugadoras aleros de baloncesto. Revista Internacional de Ciencias del Deporte. 4(2), 26-46.

\begin{tabular}{|l|ll|}
\hline \multirow{2}{*}{ Otras cualidades } & 0 & Inteligencia. \\
& 0 & Creatividad. \\
0 & Carácter. \\
& 0 & Buena visión de juego. \\
\hline Tipos de jugadora alero & 0 & Alero. \\
& 0 & Escolta (alero-base). \\
& 0 & Alero alto (ala-pívot). \\
\hline $\begin{array}{l}\text { Aspectos destacables de las mejores } \\
\text { aleros que han tenido los }\end{array}$ & 0 & Buena calidad técnico táctica. \\
entrenadores & 0 & Buena capacidad de decisión. \\
& 0 & Gran percepción del juego. \\
\hline $\begin{array}{l}\text { Funciones específicas de las } \\
\text { jugadoras aleros en sus equipos }\end{array}$ & 0 & $\begin{array}{l}\text { Tiro exterior y media distancia. } \\
\text { Acciones de 1x1. } \\
\text { Pases interiores a los pívots. }\end{array}$ \\
\hline $\begin{array}{l}\text { Participación de las aleros en el } \\
\text { ataque }\end{array}$ & 0 & Semejante al resto de las jugadoras. \\
\hline $\begin{array}{l}\text { Participación de las jugadoras aleros } \\
\text { en situaciones arriesgadas }\end{array}$ & 0 & Igual que el resto de las jugadoras. \\
\hline $\begin{array}{l}\text { Tomas de decisión de una jugadora } \\
\text { alero }\end{array}$ & 0 & $\begin{array}{l}\text { Son frecuentes y deben ser correctas en todo momento, } \\
\text { tanto a lo largo del partido como en situaciones finales, con } \\
\text { un tanteo igualado. }\end{array}$ \\
\hline
\end{tabular}

Cuadro 1. Perfil de las jugadoras aleros de Baloncesto, según los entrenadores de la Liga Femenina.

\section{Estudio 2}

\section{Características personales y deportivas de las jugadoras aleros}

Consideramos necesario obtener información sobre las jugadoras aleros y el tipo de juego que desarrollaban en la competición. Para ello planteamos los siguientes objetivos:

1. Valorar los antecedentes deportivos de cada jugadora relacionados con el Baloncesto.

2. Conocer las circunstancias deportivas que rodearon a las jugadoras en la temporada en la que se realizó el estudio.

3. Analizar qué situaciones del juego eran más motivadoras para las jugadoras; cuáles eran sus preferencias, y la forma de tomar decisiones durante los partidos.

4. Valorar qué conocimientos específicos tenía la jugadora en cuanto a la posición de la jugadora alero y a sus posibles acciones en función de las circunstancias del juego.

5. Conocer qué nivel de conocimiento poseían, relacionado con diversas tomas de decisión en determinadas situaciones de los partidos. 


\section{Resultados}

Tras la aplicación del cuestionario específico (Q.JAB), se analizaron las respuestas en función de cada uno de los correspondientes apartados. Posteriormente, realizamos las siguientes conclusiones:

\section{Características personales}

o Las jugadoras analizadas empezaron a jugar a baloncesto hacia los 10-12 años. El colegio y la familia fueron los que les motivaron a iniciarse en este deporte, y continuaron porque creían que tenían las características apropiadas, lo hacían bien y fundamentalmente porque se divertían. El número de años entrenando, con un promedio superior a diez, y el número de horas dedicadas al entrenamiento, superior a las quince horas semanales (que suponen entre 600 y 900 horas por temporada), así como la calidad de juego y la experiencia que han ido adquiriendo a lo largo de las temporadas, y la categoría y equipos a los que han pertenecido, son factores que hacen que se pueda considerar a estas jugadoras como unas baloncestistas expertas, con un gran dominio en las diferentes áreas de la percepción, decisión y ejecución (Castejón, 2002a; Ruiz Pérez, 1999; Sáenz-López, Giménez, Sierra, Ibáñez y Sánchez, 2003; Sánchez Sánchez, 2002).

o Todas las jugadoras manifestaron que tenían una buena formación técnica, táctica y física. En cuanto al conocimiento del reglamento, tres jugadoras expusieron que tenían un bajo conocimiento en esta área. Su formación psicológica fue nula.

o Las jugadoras reflejaron que dedicaban un número de horas elevado de entrenamiento, alrededor de quince horas semanales sin contar el día del partido, y todas jugaron con el puesto específico de alero.

\section{Preferencias de las jugadoras aleros}

o Jugar los partidos era la actividad que más les gustaba. Por otro lado, destacaron que, dentro del entrenamiento, la preparación física es el área que menos les motivaba.

o La competitividad, la diversión al jugar los partidos, el gusto por ganar, afán de superación, disciplina, respeto y madurez, fueron las consideraciones que realizaron las jugadoras como fuentes de motivación.

o Todas las jugadoras manifestaron que la posición de alero era la que más les gustaba porque tenían libertad de acción y porque disfrutaban en esta responsabilidad.

o Sus lugares preferentes en el campo de ataque fueron los laterales del campo de ataque, por detrás de la línea de 6,25 metros, entre la prolongación de la línea de tiros libres y la línea de fondo. 
Jiménez, A.C., Ruiz, L.M. (2006). Análisis de las tomas de decisión en la fase de ataque de las jugadoras aleros de baloncesto. Revista Internacional de Ciencias del Deporte. 4(2), 26-46.

\section{$\underline{\text { Conocimiento sobre el juego }}$}

o Las acciones de ataque más realizadas por las aleros, según su opinión, fueron las de tirar a canasta en suspensión, penetrar hacia el aro, finalizar el contraataque e ir al rebote ofensivo.

o Dejaron patente que lo que debían hacer era resolver la jugada rápidamente, realizando un tiro a canasta, una penetración o un pase. En cuanto a las variables que podían influir en las acciones realizadas cuando recibían el balón en el campo de ataque, todas las jugadoras destacaron la posición de la defensora y la distancia de ésta a la jugadora con balón, así como el tiempo y resultado del partido. También coincidieron en considerar que la acción que se elige en el partido depende también del sistema de ataque planteado por el entrenador.

o Las acciones de tirar a canasta desde detrás de la línea de 6,25 metros y penetrar a canasta mediante una situación de $1 \mathrm{x} 1$, fueron las acciones elegidas preferentemente. La opción de dar asistencias a sus compañeras también fue compartida por una mayoría.

\section{$\underline{\text { Sobre las tomas de decisión }}$}

o Las jugadoras expresaron que, en ocasiones, tomaron decisiones acertadas en situaciones límite, en que el tiempo para finalizar el partido fue mínimo, coincidiendo a su vez con un ajustado tanteo en el marcador.

o Preferían elegir ellas una acción determinada y no esperar a que el entrenador u otra compañera les indicaran lo que tenían que hacer.

o En cuanto a tomar decisiones arriesgadas, reflejaron que les gustaba porque si no, no podrían jugar a baloncesto.

o Jugadoras expertas por el número de años entrenando y los resultados conseguidos.

o Sobrevaloran la labor didáctica y humana del primer entrenador.

o Sienten gran motivación por entrenar y jugar. Les gusta tener el puesto específico de alero.

o Les gusta más atacar que defender. Lanzar a canasta es su principal objetivo, así como tomar constantes decisiones a lo largo del juego.

o Consideran determinantes para tomar una decisión la posición de la defensora, así como el tiempo y resultado parcial del partido.

o La acción más habitual de ataque es la realización de 1x1. 


\section{Estudios 3 y 4}

\section{Aspectos psicológicos de las jugadoras}

Desde un perfil psicológico, indagamos sobre los niveles de percepción del éxito de las jugadoras y sus orientaciones motivacionales (Castillo, Balaguer y Duda, 2000; Cervelló, Escartí y Balagué, 1999). También constatamos las preferencias y sus estilos de decisión, relacionadas con tres parámetros: la competencia decisional percibida, la ansiedad y agobio al decidir y el compromiso en el aprendizaje táctico decisional (Ruiz, Graupera y Sánchez, 1998; Ruiz, Navarro y Graupera, 2000).

\section{Sobre la percepción del éxito}

El punto de partida, fue conocer la motivación de logro orientadas a la tarea o al ego de las jugadoras aleros de baloncesto. Los objetivos de este estudio fueron:

1. Comprobar cuáles son los elementos que las jugadoras aleros relacionan con su percepción del éxito.

2. Conocer cuáles son las inclinaciones de las jugadoras respecto a la orientación a la tarea y hacia el ego.

Se aplicó el cuestionario de percepción del éxito (POSQ) de Roberts y Balagué (1989). Consta de 12 ítems, de los cuales seis miden la orientación de meta disposicional hacia la tarea y otros seis miden la orientación hacia el ego.

Los resultados manifestaron que:

o Todas las jugadoras aleros tuvieron la máxima puntuación en las respuestas relacionadas con la orientación a la tarea.

o Obtuvieron una alta valoración en las respuestas orientadas al ego.

o Las jugadoras aleros de esta investigación sentaron sus bases en el trabajo, el esfuerzo y afán de superación por conseguir mejorar.

o Dado su nivel de experiencia y competición que realizan, percibieron que habían conseguido resultados superiores a la mayoría de las jugadoras de baloncesto, y se consideraban entre las mejores.

Figura 1. Puntuaciones de las jugadoras en las dos dimensiones del cuestionario de percepción del éxito (Roberts y Balagué, 1999).

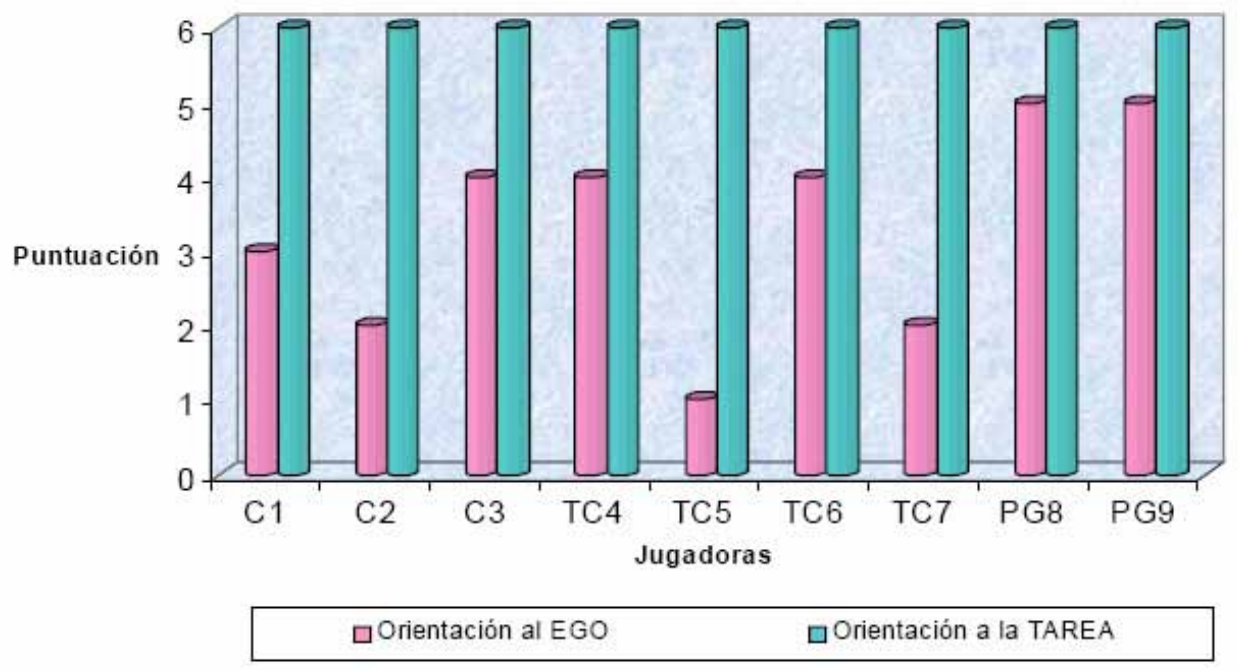




\section{Estilos de decisión de las jugadoras aleros}

Los objetivos planteados fueron:

1. Establecer el tipo de perfil decisional de las jugadoras aleros.

2. Comparar el perfil decisional de las jugadoras con otros estudios.

Se aplicó el cuestionario de estilo de decidir en el deporte (Ruiz, Sánchez y Graupera, 1998), considerando tres dimensiones que interactúan en los deportes con una alta exigencia decisional (competencia decisional, ansiedad y agobio al decidir y, la tercera, el compromiso en el aprendizaje táctico).

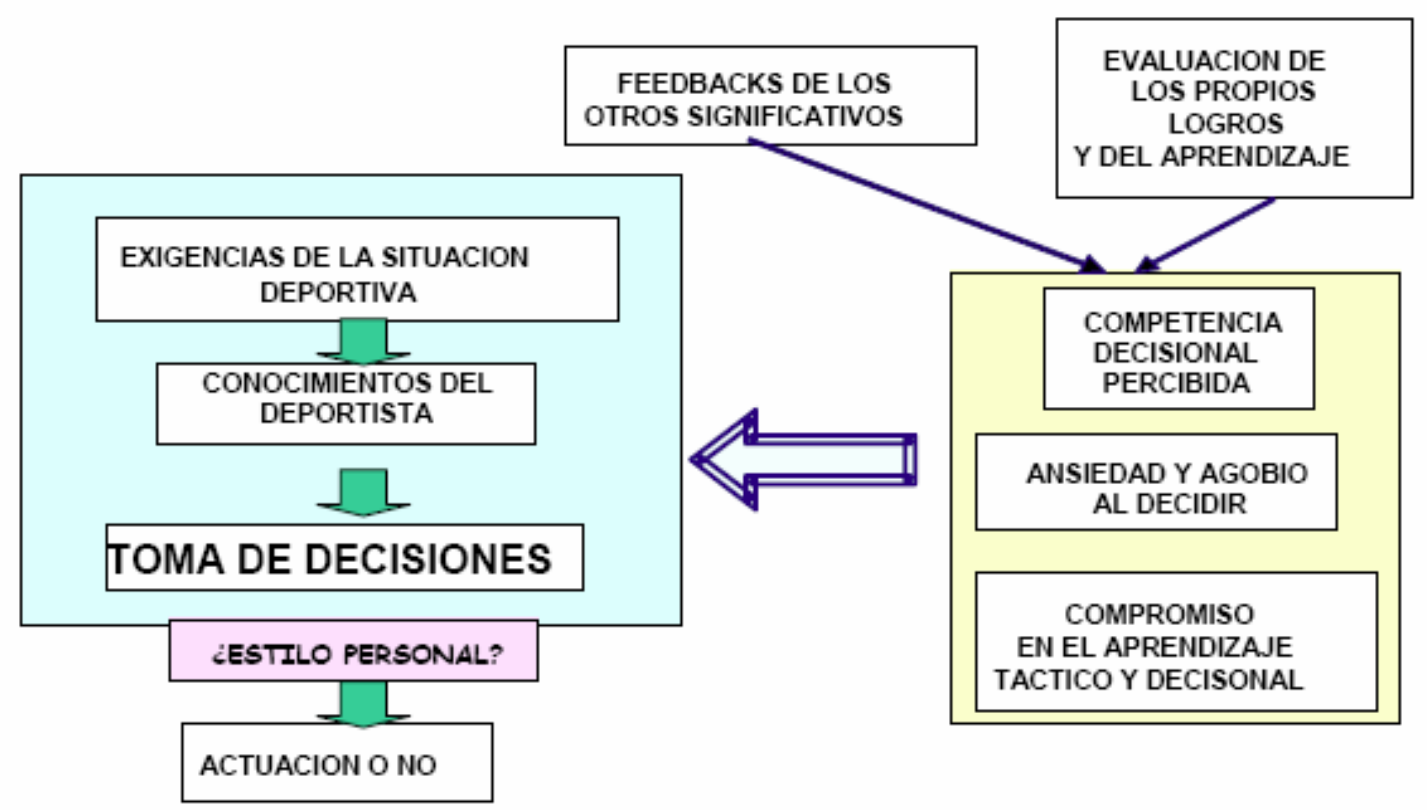

Figura 2. Modelo teórico sobre la interacción de las dimensiones que interactúan en los deportes de alta exigencia decisional (Ruiz, 2002).

Los resultados con relación a las tres dimensiones planteadas fueron los siguientes:

$\underline{\text { Respecto a la competencia decisional percibida }}$

o Los resultados fueron superiores a otras deportistas de élite.

o Manifestaron clara preferencia por tomar decisiones.

o La motivación por tomar decisiones, va unida a la elevada confianza que tenían en sí mismas.

En cuanto a la ansiedad y agobio al decidir

o La puntuación es claramente más baja que la de otras deportistas.

o No manifestaron ansiedad al decidir bajo presión. Asumían con valentía las consecuencias de sus errores. 
Jiménez, A.C., Ruiz, L.M. (2006). Análisis de las tomas de decisión en la fase de ataque de las jugadoras aleros de baloncesto. Revista Internacional de Ciencias del Deporte. 4(2), 26-46.

\section{$\underline{\text { Compromiso en el aprendizaje táctico-decisional }}$}

o No hay diferencias notables con otros deportistas.

o Este resultado nos hace pensar que se debe a un alto grado de libertad con relación a las pautas estratégicas marcadas por el entrenador.

\section{Estudio 5}

\section{Decisiones y acciones de las jugadoras aleros durante la competición}

Consideramos necesario realizar el análisis de las acciones realizadas por las jugadoras, durante los partidos, desde el momento en que recibieron el balón en la fase de ataque, así como las variables que afectaban a dichas acciones realizadas, fueron motivo de interés en esta investigación. Se analizaron 34 partidos (todos los jugados en el campo local a lo largo de una temporada).

Los objetivos específicos planteados fueron:

1. Comprobar las condiciones en las que se realizaron las secuencias de decisiónacción de las jugadoras, con relación al tiempo y resultado del partido; la posición en donde recibieron; la posición y distancia de la defensora; si las defensoras realizaron alguna ayuda defensiva, o si el árbitro interrumpió el juego.

2. Analizar las acciones de ataque que realizaron las jugadoras aleros de baloncesto durante los partidos, cuando recibieron el balón en el campo de ataque.

3. Comparar los resultados de las acciones registradas con los resultados obtenidos en los otros estudios de la investigación.

El procedimiento para analizar las acciones realizadas por las jugadoras durante los partidos, siguió determinados pasos:

- Realización de un estudio piloto.

- Selección de las variables que consideramos que podían influir en las jugadoras, y de las posibles acciones técnicas y tácticas que podían realizar cuando recibían el balón, con la intención de que nos pudiera ayudar a confeccionar dicha hoja de análisis de tomas de decisión.

- Confección de una hoja de registro provisional y definición de categorías. Validación por expertos.

- Elaboración definitiva de la Hoja de Análisis de Tomas de Decisión (HATD). 


\section{Resultados}

$\underline{\text { Sobre las variables que pueden influir en las acciones de las jugadoras }}$

o El número de ataques realizados por partido, en consonancia con el número de minutos jugados de las jugadoras, ha sido proporcionado. La participación en ataque de dos jugadoras fue mayor que la del resto de jugadoras.

o Las áreas de recepción del balón son los laterales del campo, por encima de la prolongación de los tiros libres. Esta zona difiere a las zonas de preferencias por las jugadoras analizadas, aunque consideramos que no tiene mayor relevancia.

o Las jugadoras recibieron el balón en movimiento el mayor número de ocasiones (64,7\%), y además orientaron su cuerpo hacia el aro nada más recibir en el 93,6 \% de las veces que recibieron el balón.

o En el mayor número de ocasiones (86\%), las jugadoras tardaron menos de tres segundos en realizar una acción, una vez que recibieron el balón.

o Con respecto a la posición de triple amenaza, sólo el 23\% de las veces adoptaron esta posición. Habría que cuestionarse la importancia de adoptar esta posición, si bien es cierto que cuando se recibe en movimiento, como la mayor parte de las ocasiones, es difícil adoptar esta posición.

o En un elevado número de ocasiones, las defensoras se encontraban a la distancia aproximada de un metro, o incluso más alejada. Este aspecto justifica que las jugadoras tomaran como primera opción realizar un pase. Es cierto que podrían haber tirado, pero parece ser que tuvieron en cuenta si sus recepciones de balón se hicieron al inicio de la fase de ataque. En algunos ataques hubo la presencia de ayudas defensivas por parte de otras defensoras que provocaron los correspondientes ajustes en las decisiones y acciones de las jugadoras.

o Las interrupciones arbitrales fueron escasas. Sólo mencionar que el 4,2 \% los colegiados pitaron una falta a favor de la jugadora con balón, con las repercusiones positivas que tuvo para la jugadora y para el equipo.

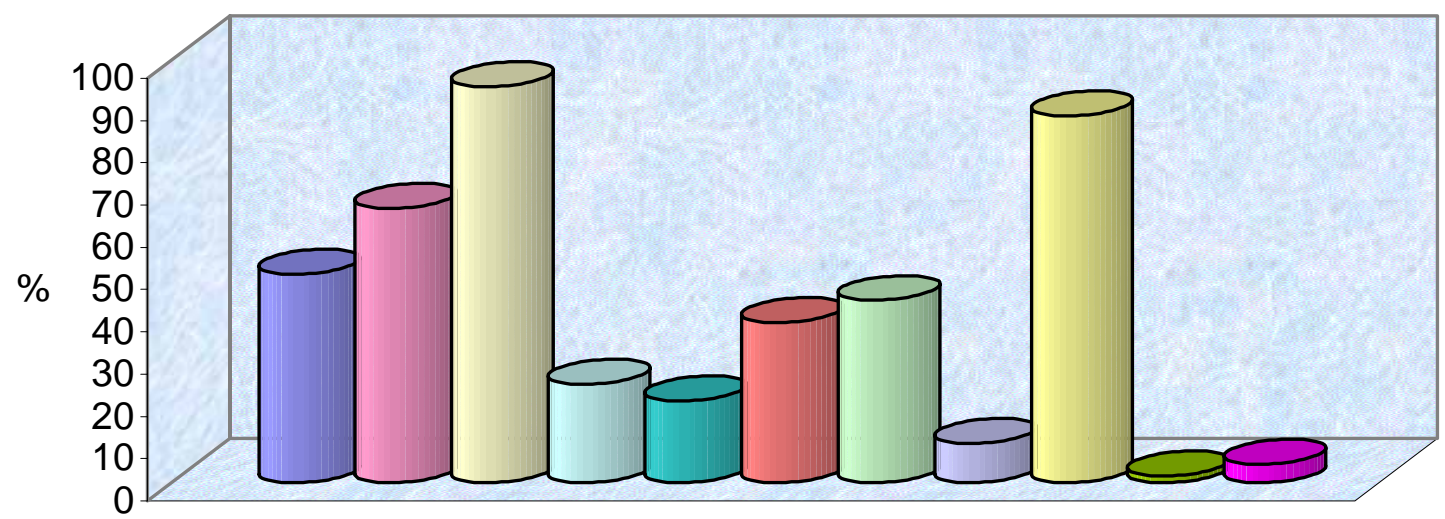

\begin{tabular}{|lll|}
\hline$\square$ Áreas más frecuentes: 7 y 9 & $\square$ Recibe en movimiento & $\square$ Orienta su cuerpo al aro \\
$\square$ Posición triple amenaza & $\square$ Defensa presionante & $\square$ Defensa posición media \\
$\begin{array}{l}\square \text { Defensa alejada } \\
\square \text { Infracción de la alero }\end{array}$ & $\square$ Ayudas defensivas & $\square$ Tiempo menor 3 seg. \\
\hline
\end{tabular}

Figura 3. Porcentajes sobre las variables que pueden influir en las acciones de las jugadoras. 


\section{$\underline{\text { Sobre las acciones realizadas }}$}

o La acción botar, las jugadoras aleros la utilizaron con cierta frecuencia (34,4\%), pero debemos indicar que, aunque en muchas ocasiones, iniciaron su primera acción con un bote en el mismo lugar sin desplazarse, aunque esto no impidió realizar una segunda acción en buenas condiciones. Seguimos planteándonos la cuestión sobre el uso de este tipo de bote que, aunque consideramos que en el caso de estas jugadoras no repercutió negativamente en sus acciones posteriores, sería más conveniente adoptar en más ocasiones la posición de triple amenaza, en sustitución de dicho bote.

o Con relación a la acción de pasar, hubo predominio de los pases exteriores respecto a los interiores. La importancia de repartir el juego, sobre todo en los momentos iniciales de la fase de ataque, consideramos que fue lo que provocó fundamentalmente la realización de los pases exteriores.

o Las jugadoras realizaron un total de 58 asistencias, entendiéndolas como el grado de excelencia en la realización de un pase.

o Las pérdidas de balón, consideramos que fueron bajas (2,5\%), y quizá sea ésta una apreciación importante, a favor del nivel de calidad de las jugadoras analizadas.

o Respecto a los lanzamientos a canasta realizados, en esta investigación estudiamos, por un lado los tiros de dos puntos y los de tres realizados en suspensión desde áreas próximas al lugar de recepción del balón. Por otro lado, analizamos las entradas a canasta, y las situaciones finales de contraataque. No recogimos las acciones de tiros libres realizados por las jugadoras. Por este motivo, no se puede apreciar al completo el porcentaje total de tiros a canasta, pero sí podemos afirmar que los porcentajes de lanzamientos a canasta son significativos y garantizan la participación activa de las jugadoras aleros en la fase de ataque (aunque hubo diferencias notables entre unas y otras jugadoras). La suma de los tiros de dos y tres puntos y las entradas a canasta realizadas fue de 719 , que supone el $31,7 \%$ del total de acciones realizadas.

o Queremos destacar el porcentaje de aciertos en comparación con el número de lanzamientos realizados, que en este caso corresponde al 28,8\%, teniendo en cuenta que en este apartado no se contabilizaron las canastas conseguidas en los tiros libres y las terminaciones de contraataque, puesto que no eran nuestro objetivo.

o Respecto a las entradas a canasta, queremos considerar que el mayor número de las que se realizaron fueron directas al aro, pero también tuvieron representación las entradas a canasta con parada y tiro, y por último, fueron muy escasas aquellas que se realizaron con parada, finta y tiro.

o Resaltamos la presencia de las fintas sobre todo al inicio de la acción, con la intención de engañar a la defensora. 
Jiménez, A.C., Ruiz, L.M. (2006). Análisis de las tomas de decisión en la fase de ataque de las jugadoras aleros de baloncesto. Revista Internacional de Ciencias del Deporte. 4(2), 26-46.

o En cuanto al contraataque, como acción en donde participó la jugadora en los momentos finales de éste (en 112 ocasiones), sólo queremos destacar la importancia de las canastas realizadas en estas condiciones que exige una gran rapidez perceptiva y de decisión.

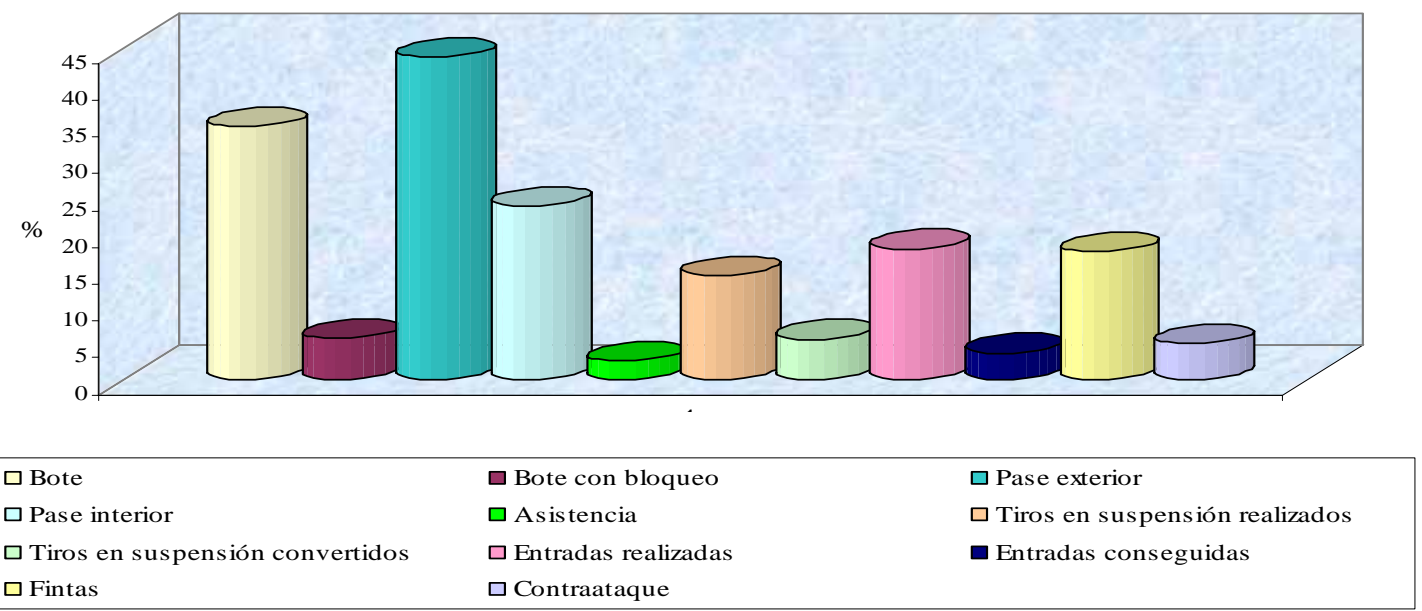

Figura 4. Porcentaje sobre las acciones realizadas por las jugadoras aleros.

\section{Estudio 6}

\section{El pensamiento de las jugadoras aleros sobre sus tomas de decisión y sus acciones}

Las interpretaciones obtenidas por las jugadoras, respecto a sus propias acciones durante los partidos, a través de protocolos verbales evocadores realizados en un plazo mínimo de tiempo después de transcurridos dichos partidos, aportaron datos muy esclarecedores en el conjunto de la investigación. Se realizaron un total de 18 entrevistas.

Los objetivos fueron los siguientes:

1. Conocer los razonamientos de las jugadoras sobre su toma de decisión.

2. Considerar sus pensamientos y percepciones previos al partido.

3. Relacionar sus pensamientos antes de los partidos, con sus actuaciones posteriores en el transcurso de dichos partidos.

4. Conocer qué elementos tomaban como referencia las jugadoras aleros al recibir el balón, en las determinadas situaciones de ataque que se les presentaba. 
Jiménez, A.C., Ruiz, L.M. (2006). Análisis de las tomas de decisión en la fase de ataque de las jugadoras aleros de baloncesto. Revista Internacional de Ciencias del Deporte. 4(2), 26-46.

El instrumento utilizado fue una entrevista semiestructurada, partiendo de un guión establecido basado en temas generales concretos, y a partir de ahí fomentamos la expresión libre de la jugadora.

El procedimiento seguido en esta investigación fue la siguiente:

- Tras la filmación y el análisis del partido, se realizó una selección de las secuencias de las jugadoras en la fase de ataque. Las más representativas, en torno al 50\%.

- Previo acuerdo con la jugadora, en un plazo máximo de 48 horas se realizaron las entrevistas.

- De cada secuencia presentada a las jugadoras se le ofreció información en torno al tiempo y tanteo, la zona de recepción del balón, si su defensa estaba cerca o lejos, etc.).

- La jugadora observaba la proyección de cada una de las acciones presentadas, e inmediatamente describía verbalmente la situación respondiendo a las cuestiones planteadas en torno a la misma.

- Todas las respuestas y comentarios de las secuencias de ataque seleccionadas, al igual que el resto de la entrevista, quedaron grabadas y transcritas. Posteriormente se procedió a una lectura detenida. Realizamos una extracción de aquellos aspectos que considerábamos significativos para el problema planteado y después establecimos los grandes temas.

\section{Resultados}

\section{$\underline{\text { Respecto a las percepciones previas al partido jugado }}$}

o Las jugadoras tenían un conocimiento muy preciso sobre el equipo con el que se enfrentaban así como la clasificación que tenían en la competición.

o Total confianza en que iban a ganar el partido.

o Valoraron su estado físico y anímico, así como la calidad con la que habían entrenado durante la semana.

o Tenían una clara percepción de cuál iba a ser su participación en le partido. 


\section{$\underline{\text { Respecto a las acciones realizadas }}$}

1. Gran capacidad para describir y justificar las acciones realizadas.

2. El tiempo para finalizar el partido, incluso el tiempo para finalizar cada fase de ataque, así como el tanteo en el marcador fueron variables a tener en cuenta.

3. El espacio utilizado venía determinado por los lugares de la recepción del balón. En ocasiones las jugadoras indicaron que no podían haber realizado un tipo de acción porque sus propias compañeras no dejaban espacio en un momento concreto.

4. Se fijaron en la posición de la defensa y en la distancia que tenían respecto a la jugadora con balón. Incluso en sus características físicas y nivel de experiencia, en algunos casos. El resto de defensoras también lo tuvieron en cuenta, sobre todo después de haber iniciado la acción.

5. Los sistemas de ataque fueron patrones que mejoraban las acciones de dichas jugadoras. Ninguna de ellas planteó que les perjudicara.

6. Con relación a sus decisiones tomadas durante el partido, la mayoría de las jugadoras consideraron que fueron acertadas. En caso de error, reconocieron perfectamente cuál había sido el problema.

7. Existieron situaciones de decisiones arriesgadas. Observamos que las jugadoras resolvieron la situación sin dudas.

8. En alguna ocasión las jugadoras abordaron la importancia de la sincronización con otras compañeras para obtener resultados favorables.

9. En dos jugadoras se aprecia que el nivel de riesgo de sus decisiones, durante los partidos, tiene un alto grado de subjetividad. Es decir, ellas tuvieron una escasa participación durante los partidos y manifestaron que cuando salían a jugar, el riesgo de lanzar a canasta y no conseguir encestar podría significar no seguir jugando, y ésta era una variable a tener en cuenta.

10. Sólo una jugadora manifestó que en función de cómo iba su equipo, con su actuación decidía incrementar el ritmo del partido o no.

11. En cuanto a los árbitros, las jugadoras no consideraron que éste fuera determinante en sus decisiones. Incluso, una jugadora indicó que podía actuar diferente, en beneficio propio, según la decisión puntual del árbitro. 


\section{CONCLUSIONES}

1. Las jugadoras aleros analizadas eran expertas y, como tal, tenían un elevado nivel cualitativo que les permitía tener una gran calidad en lo que percibían durante el partido, al igual que en sus tomas de decisión y sus ejecuciones.

2. Los espacios utilizados en el campo de ataque fueron los laterales, por encima de la línea de 6,25 metros. Recibieron balón en movimiento. Orientaron su cuerpo hacia el aro inmediatamente. Iniciaron sus acciones en menos de tres segundos. Manifestaron tener gran número de recursos para afrontar las decisiones y acciones que les planteaba el juego. Resolvieron con facilidad situaciones de 1x1 y 2x2.

3. Fueron conscientes de las numerosas tomas de decisión durante los partidos, y éstas fueron un elemento motivador en sus responsabilidades.

4. Gran percepción del éxito y confianza en sí mismas. Los valores en las orientaciones a la tarea y al ego fueron elevados.

5. Sus estilos al decidir en le deporte fueron muy óptimos para afrontar la competición de baloncesto con éxito.

6. La posición y la distancia de las defensoras fueron determinantes en las acciones elegidas por las jugadoras.

7. El tiempo para finalizar la fase de ataque y el partido, así como el resultado de éste fueron referentes directos en las tomas de decisión.

8. El estado físico, anímico y el grado de satisfacción por el entrenamiento de la semana anterior al partido, les aportó mayor o menor confianza.

9. Los sistemas de ataque fueron un referente que les guiaba, pero no condicionaba sus acciones.

10. En sus reflexiones sobre las decisiones y acciones realizadas, manifestaron gran conocimiento sobre el partido y un elevado optimismo en cuanto a las posibilidades de ganar. Discriminaron errores de decisión de los de ejecución. Fueron capaces de ofrecer alternativas a sus propias decisiones.

11. Considerado el grupo de jugadoras analizado como expertas, se aprecian claramente dos perfiles de jugadoras de baloncesto de alta competición, valorando diversos matices cualitativos y cuantitativos que distingue un grupo de otro. Por tanto, definimos a un grupo de jugadoras expertas y otro en que las jugadoras son excelentes, y se caracterizan por ser:

- $\quad$ Más eficientes.

- Más resolutivas.

- Por tener control sobre otras variables del juego tales como el ritmo del partido, las propias decisiones de los árbitros, la coordinación con sus compañeras y la rapidez y características de la defensora.

12. Las tomas de decisión en baloncesto tienen la suficiente importancia como para ser tratada y entrenada minuciosamente por los jugadores y entrenadores. 


\section{Bibliografía}

Abernethy, B. (1987). Selective attention in fast ball sports II: experts-novice differences. The Australian J ournal of Science and Medicine in Sport, 19(4), 7-16.

Abernethy, B. (1989). Expert-novice difference in perception: How expert does the expert have to be? Canadian Journal of Sport Science, 14(1), 27-30.

Buscá, B., Pont, J., Artero, V. y Riera, J. (1996). Propuesta de análisis de la táctica individual ofensiva en el fútbol. Apunts. Educación Física y Deportes, 43, 63-74.

Cárdenas, D. y Pintor, D. (2001). La iniciación al baloncesto en el medio escolar. En F. Ruiz, A. García y A.J. Casimiro (Eds.), La iniciación deportiva basada en los deportes colectivos. Madrid: Gymnos.

Castejón, F.J. (2002a). Decisión estratégica y decisión táctica. Similitudes, diferencias y aplicaciones en el deporte. Revista del Entrenamiento Deportivo, 16(4), 31-39.

Castejón, F.J. (2002b). Expertos y novatos en el proceso de enseñanza aprendizaje y su implicación en la iniciación deportiva. Habilidad Motriz, 20, 13-27.

Castejón, F.J., Aguado, R., Cabrera, M.L., García, E., Gismero, M.L., Rodríguez, J.J. y Romero, M.J. (2004). La toma de decisión estratégica en entrenadores. Diferencias entre expertos y novatos. Revista de entrenamiento deportivo.

Castejón, F.J. y López, V. (2000). Solución mental y solución motriz en la iniciación a los deportes colectivos en la educación primaria. Apunts. Educación Física y Deportes, 61, 37-47.

Castillo, I., Balaguer, I. y Duda, J.L. (2000). Las orientaciones de meta y los motivos de práctica deportiva en los jóvenes deportistas valencianos escolarizados. Revista de Psicología del Deporte, 9(1-2), 37-50.

Cervelló, E., Escartí, A. y Balagué, G. (1999). Relaciones entre la orientación de meta disposicional y la satisfacción con los resultados deportivos, las creencias sobre las causas de éxito en el deporte y la diversión con la práctica deportiva. Revista de Psicología del Deporte, 8(1), 7-19.

Chelladurai, P. (1991). Estilos de toma de decisión en el entrenamiento. En J.M. Williams (Ed.), Psicología aplicada al deporte (pp. 178-192). Madrid: Biblioteca Nueva.

Chelladurai, P. y Quek, C.B. (1995). Decision style choices of high school basketball coaches: The effects of situacional and coach characteristics. Journal of Sport Behavior, 18(2), 91108.

Dupré, P. (2001). La prise de décision. EPS, 289, 77-81.

Fernández, E., Sánchez, F. y Fernández-Quevedo, C. (2002). Estudio de las habilidades de resolución de problemas en el fútbol sala desde una perspectiva de género. Investigación de Ciencias del Deporte, 35, 135-179.

French, K.E., Nevett, M.E., Spurgeon, J.H., Graham, K.C., Rink, J.E. y McPherson, S.L. (1996). Knowledge representation and problem solution in expert and novice youth baseball players. Research Quarterly for Exercise and Sport, 67(4), 386-395.

French, K.E., Spurgeon, J.H. y Nevett, M.E. (1995). Expert-novice differences in cognitive and skill execution components of youth baseball performance. Research Quarterly for Exercise and Sport, 66(3), 194-201.

French, K.E. y Thomas, J.R. (1987). The relation of knowledge development to children's basketball performance. Journal of Sport Psychology, 9, 15-32.

Giménez, F.J. y Sáenz-López, P. (2000). Aspectos teóricos y prácticos de la iniciación al baloncesto. Huelva: Diputación de Huelva. 
Jiménez, A.C., Ruiz, L.M. (2006). Análisis de las tomas de decisión en la fase de ataque de las jugadoras aleros de baloncesto. Revista Internacional de Ciencias del Deporte. 4(2), 26-46.

http://www.cafyd.com/REVISTA/art3n4a06.pdf

Giménez, F.J. y Sáenz-López, P. (2002). El baloncesto en la escuela: ¿De la base a la élite? En S.J. I báñez y M. Macías (Eds.), Novos horizontes para o treino do basketebol. Cruz Quebrada: Faculdade de Motricidade Humana.

Hernández, J. (2001). Ejercicios de 1 contra 1. Valencia: Promolibro.

Ibáñez, S.J. (2000). La iniciación al juego colectivo a través de la ocupación de espacios libres. En J. Giménez y P. Sáenz-López (Eds.), Análisis de la iniciación al Baloncesto (pp. 71-102). Huelva: Diputación de Huelva.

Iglesias, D., Moreno, P., Ramos, L.A., Fuentes, J.P., Julián, J.A. y Del Villar, F. (2002). Un modelo para el análisis de los procesos cognitivos implicados en la toma de decisiones en deportes colectivos. Revista de Entrenamiento Deportivo, 16(15), 9-14.

Lane, I.M. y Damiano, P.L. (1994). Determining decision-making effectiveness using NCAA basketball tournament result. Journal of Sport Behavior, 17(2), 79-86.

Lorenzo, A. (2003). Estudio del pensamiento de los entrenadores sobre el proceso de detección de talentos en baloncesto. Motricidad, 10, 23-51.

Moreno, P., Fuentes, J.P., Del Villar, F., Iglesias, D. y Julián, J.A. (2003). Estudio de los procesos cognitivos desarrollados por el deportista durante la toma de decisiones. Apunts. Educación Física y Deportes, 73, 24-29.

Olivera, J. (2001). De los juegos colectivos a los deportes de equipo. Apunts. Educación Física y Deportes, 64, 3-4.

Refoyo, I. (2001). La decisión táctica de juego y su relación con la respuesta biológica de los jugadores. Una aplicación al baloncesto como deporte de equipo. Unpublished Tesis Doctoral no publicada, Universidad Complutense de Madrid, Madrid.

Ribas, J. y Fraile, C. (2003). Predicción de puestos específicos de jugadores en etapas de formación. En S. I báñez y M. Macías (Eds.), Propuestas para la mejora en el proceso de formación y rendimiento en baloncesto. Cáceres: Universidad de Extremadura.

Ripoll, H. (1988). Analysis of visual scanning patterns of volley ball players in a problem solving task. International J ournal of Sport Psychology, 19, 9-25.

Ruiz, L.M. (2002). Decidir en el deporte: Un proceso personal entre el conocimiento y la confianza. II Congreso de Ciencias del Deporte (Actas no publicadas).

Ruiz, L.M. y Arruza, J. (2005). El proceso de toma de decisiones en el deporte. Clave de la eficiencia y el rendimiento óptimo. Barcelona: Paidós.

Ruiz, L.M., Graupera, J.L. y Navarro, F. (2000). Construcción, análisis psicométrico y tipificación de un cuestionario de toma de decisión en el deporte. Madrid: Centro de Alto Rendimiento y de Investigación en el Deporte. Consejo Superior de Deportes. CARICD.

Ruiz, L.M., Graupera, J.L. y Sánchez, F. (1998). La toma de decisión en el deporte. (Documento no publicado).

Ruiz, L.M., Navarro, F. y Graupera, J.L. (2000). Construcción, análisis psicométrico y tipificación de un cuestionario de toma de decisión en el deporte. Madrid: Consejo Superior de Deportes.

Ruiz Pérez, L.M. (1999). Rendimiento deportivo, optimización y excelencia en el deporte. Revista de Psicología del Deporte, 8(2), 235-248.

Sáenz-López, P. (2000). La iniciación del baloncesto a través del juego. En F.J. Giménez y P. Sáenz-López (Eds.), Análisis de la iniciación al baloncesto (pp. 115-129). Huelva: Diputación de Huelva.

Sáenz-López, P., Giménez, F.J., Sierra, A., Ibáñez, S. y Sánchez, M. (2003). La formación del jugador experto de baloncesto en España. En S. Ibáñez y M. Macías (Eds.), Propuestas para la mejora en el proceso de formación y rendimiento en baloncesto. II Congreso I bérico de Baloncesto. Cáceres: Universidad de Extremadura.

Sánchez, M. (2002). El proceso de llegar a ser experto en baloncesto: Un enfoque psicosocial. Universidad de Castilla-La Mancha, Toledo. 
Tavares, F. (2002). Processos cognitivos e a performance no basquetebol. Elementos para a análise do comportamento de decisao táctica, do conhecimento do jogo e da execuçao das acçoes defensivas no jovem jogador. En S.J. Ibáñez y M. Macías (Eds.), Novos horizontes para o treino do basquetebol (pp. 155-178). Cruz Quebrada: Facultad de Motricidade Humana.

Temprado, J.J. (1989). Prise de decision en sport: modalités d'etudes et données actuelles. STAPS, 10(19), 53-67.

Temprado, J.J. (1992). Les apprentisages dècisionnels en EPS. En J.P. Famose., P.H. Fleurance y Y. Touchard (Eds.), I'apprentisage moteur. Rôle de representations. Paris: EPS.

Temprado, J.J. y Alain, C. (1999). Elementos para el análisis del comportamiento de decisión del defensor en los deportes de raqueta. En J.P. Famose (Ed.), Cognición y rendimiento motor (pp. 45-64). Barcelona: Inde.

Temprado, J.J. y Famose, J.P. (1999). Análisis de la dificultad en el tratamiento de información y descripción de las tareas motrices. En J.P. Famose (Ed.), Cognición y rendimiento motor (pp. 177-195). Barcelona: Inde.

Tenenbaum, G., Yuval, R., Elbaz, G., Bar-Eli, M. y Weinberg, R. (1993). The relatioship between cognitive characteristics and decision making. Canadian Journal of Applied Physiology, 18(1), 48-62.

Thomas, J.R., French, K.E. y Humphries, C.A. (1986). Knowledge development and sport skill performance: directions for motor behavior research. Journal of Sport Psychology, 8, 259-272.

Ticó, J. (2002). Tareas deportivas en los deportes colectivos: Una aplicación al baloncesto. En S.J. I báñez y M. Macías (Eds.), Novos horizontes para o treino do basketebol. Cruz Quebrada: Faculdade de Motricidade Humana. 